\title{
Trends of skin blood flow response signals for early pressure ulcer evaluation
}

\begin{abstract}
Pressure Ulcer (PU) or pressure sore is localized injury to the skin or underlying tissue or both that occur over a bony prominence when a patient is subjected to direct pressure for a lengthy period. Research have shown that skin blood flow response (SBFR) is a useful signal in PU evaluation due to its ability to determine ischemic stress response. In previous animal study, peak reactive hyperaemia $(\mathrm{RH})$, a feature of SBFR has been measured for three loading-unloading cycles to indicate tissue recovery. However, in that study, peak SBFR feature cannot discriminate between tissue injury and tissue recovery effectively for short recovery time. In this study, the potential use of other SBFR signal features including time domain features i.e time to peak and frequency domain features i.e peak of power spectrum and total of power spectrum are investigated. The results show that for the samples taken, peak RH and area under the curve are both effective at discriminating between short of insufficient recovery time while other investigated features are ineffective.
\end{abstract}

Keyword: Pressure ulcer; Pressure sore; Skin blood flow response; Reactive hyperaemia; Time domain features; Frequency domain features; Peak SBFR; Area under the curve; Time to peak; Peak of power spectrum; Total power spectrum 